\title{
ESTRUCTURACIÓN DEL PRECIO DE UN MENÚ
}

\author{
Raúl Baldeón Dávalos ${ }^{1}$
}

\section{RESUMEN}

El presente trabajo propone un procedimiento claro y sencillo para establecer el costo de producción y el precio de venta de un menú.

Para establecer el precio se aplicó mecanismos de cálculo tales como, agregando un porcentaje a los costos unitarios totales y mediante un modelo de maximización de utilidades. Sin embargo será el propietario del negocio quien determine cual método aplicar dada la estructura de sus costos y el tamaño del negocio.

Se presenta un ejemplo aplicado a una microempresa dedicada a la producción y venta de sánduches, refrescos y aguas aromáticas.

\section{INTRODUCCIÓN}

En el Ecuador las pequeñas empresas dedicadas a la prestación de servicios, tales como los Delicatessen, generalmente no funcionan como empresas organizadas, tales como sociedades, sino como empresas personales.

En estos negocios normalmente se determina el precio de venta de sus productos al "ojo", por cuanto no se lleva un adecuado registro de costos y gastos para establecer el costo de producción.

Además no solamente el precio de venta, determina la competencia o no de una empresa, también se debe considerar otros aspectos como: proveedores, competidores potenciales, compradores y sustitutos.

\footnotetext{
${ }^{1}$ Facultad de Ciencias de la Ingeniería, Universidad Tecnológica Equinoccial, Av. Occidental y Mariana de Jesús. Quito, Ecuador.rfbaldeon@ute.edu.ec
} 
El objetivo de esta investigación es determinar que la falta de procedimientos técnicos por parte de microempresas dedicadas a la producción de estas actividades, hace necesario establecer un mecanismo para el cálculo de los costos en la producción que ayude a las personas dedicadas a esta actividad a que puedan establecer un precio real con el que puedan vender sus productos y servicios generando una utilidad razonable para que la empresa se mantenga en el mercado y tengan posibilidades de crecimiento.

\section{METODOLOGÍA}

\section{Formas de calcular el precio}

Cuando hay mayor exigencia, competencia, y menores márgenes de ganancia, la correcta fijación de precios es esencial. Así las cosas, es necesario ser mucho más precisos y profesionales a la hora de calcular costos y fijar precios, porque errores de cálculo de unos pocos centavos pueden costar muy caro y constituir la diferencia entre crecer, sobrevivir o sucumbir en el intento.

Es necesario calcular en primera instancia el costo de producción de cada uno de los productos que se elaboran, en este caso los materiales directos, la mano de obra directa y los costos indirectos de fabricación.

Luego establecer el cálculo del precio de acuerdo a las dos modalidades mencionadas:

- Agregando un porcentaje a los costos unitarios totales

- Mediante un modelo de maximización de utilidades

\section{Agregando un porcentaje a los costos unitarios totales:}

En este caso se determina un margen, el cual puede ser sobre los precios o sobre los costos.

\section{Si el margen es sobre el precio unitario de venta, p:}

$\mathrm{p}=\mathrm{Kp}+\mathrm{c}$

En donde 
$\mathrm{P}=$ precio unitario de venta

$\mathrm{K}=$ margen sobre el precio

$\mathrm{c}=$ costo unitario

A partir de (1.1) se obtiene:

$p=\frac{c}{1-K}$

Si el margen es sobre el costo unitario:

$\mathrm{P}=\mathrm{c}+\mathrm{mc}$
$\mathrm{P}=\mathrm{c}(1+\mathrm{m})$

En donde m es el margen sobre los costos

\section{Mediante un modelo de maximización de utilidades}

Exige llevar a cabo un análisis estadístico que permita definir, en cada caso en particular, la expresión matemática correspondiente a la función de demanda, que puede ser del siguiente tipo:

$$
\begin{aligned}
& \mathrm{Q}=\mathrm{ap}+\mathrm{bY}+\mathrm{cP}+\mathrm{dPu} \\
& \text { En donde: } \\
& \mathrm{Q}=\text { cantidad producida } \\
& \mathrm{P}=\text { precio unitario de venta } \\
& \mathrm{Y}=\text { ingreso o renta del consumidor per cápita } \\
& \mathrm{P}=\text { Población } \\
& \mathrm{Pu}=\text { gasto en publicidad }
\end{aligned}
$$

Además es necesario conocer la función de costos totales, C, correspondiente a las cantidades que pueden producirse en cada periodo

Esta función puede ser de la forma:

$\mathrm{C}=\mathrm{vQ}+\mathrm{F}$

En donde:

$\mathrm{C}=$ costo total

$\mathrm{v}=$ costo variable unitario 


$$
\begin{aligned}
& \mathrm{Q}=\text { producción } \\
& \mathrm{F}=\text { costos fijos }
\end{aligned}
$$

Para un período dado se pronostican $\mathrm{Y}, \mathrm{P}$ y $\mathrm{Pu}$, lo que implica que en dicho periodo la expresión se convierte en

$$
\mathrm{Q}=\mathrm{ap}+\mathrm{K}
$$

En donde K es una constante

La función utilidad se define como sigue:

$$
\mathrm{U}=\mathrm{I}-\mathrm{C}
$$

En donde:

$$
\begin{aligned}
& \mathrm{I}=\text { ingresos } \\
& \mathrm{C}=\text { costos }
\end{aligned}
$$

$$
\text { Pero: } \quad \mathrm{I}=\mathrm{Qp}
$$

$\mathrm{Al}$ reemplazar (1.5) y (1.8) en (1.7):

$$
\begin{aligned}
& \mathrm{U}=\mathrm{Qp}-(\mathrm{vQ}+\mathrm{F}) \\
& \mathrm{U}=\mathrm{Qp}-\mathrm{vQ}-\mathrm{F}
\end{aligned}
$$

Al reemplazar (1.6) en (1.9):

$$
\begin{aligned}
& \mathrm{U}=(\mathrm{ap}+\mathrm{K}) \mathrm{p}-\mathrm{v}(\mathrm{ap}+\mathrm{K})-\mathrm{F} \\
& \mathrm{U}=\mathrm{ap} \mathrm{p}^{2}+\mathrm{Kp}-\mathrm{vap}-\mathrm{vK}-\mathrm{F}
\end{aligned}
$$

El precio que maximiza esta función se obtiene al igualar a cero su derivada con respecto a p:

$$
\begin{aligned}
& \frac{d U}{d p}=2 a p+k-v a=0 \\
& p=\frac{v a-K}{2 a}
\end{aligned}
$$

El cálculo del precio de venta, aplicado a un solo producto, por ejemplo el sánduche entero, se inicia con los datos indicados en la tabla 1 . 


\section{RESULTADOS}

Para el primer método de cálculo, tomando como referencia los datos de la tabla 1, se establece las siguientes condiciones; si el costo unitario de un sánduche entero es de $\$ 5.64$ ¿cuál debe ser el precio de venta si el criterio es tener un margen sobre los costos de 30\%? ¿A cuánto equivale este margen si se expresa en términos del precio?

Tabla 1. Costos involucrados en la elaboración de un sánduche entero.

\begin{tabular}{lcccc}
\hline & MATERIA & MANO DE & GIF & COSTO \\
PRODUCTO & PRIMA & OBRA & & TOTAL ELAB.
\end{tabular}

\section{SÁNDUCHE ENTERO}

\begin{tabular}{|c|c|c|c|c|}
\hline Pan Baguette entero & 1,44 & & & 1,44 \\
\hline Embutidos varios & 1,92 & & & 1,92 \\
\hline Queso maduro & 0,96 & & & 0,96 \\
\hline Champiñones & 0,60 & & & 0,60 \\
\hline Mayonesa & 0,24 & & & 0,24 \\
\hline Tomate & 0,12 & & & 0,12 \\
\hline Lechuga & 0,06 & & & 0,06 \\
\hline \multirow{3}{*}{ Elaboración y despacho } & & & & - \\
\hline & \multirow{2}{*}{\multicolumn{2}{|c|}{0,24}} & & 0,24 \\
\hline & & & & - \\
\hline Fundas & & & 0,024 & 0,02 \\
\hline Servilletas & & & 0,012 & 0,01 \\
\hline Luz & & & 0,012 & 0,01 \\
\hline Depre. Cortadora y Otros & & & 0,012 & 0,01 \\
\hline TOTAL & 5,34 & 0,24 & 0,06 & 5,64 \\
\hline
\end{tabular}

Solución:

Precio de venta:

$\mathrm{P}=\mathrm{c}(1+\mathrm{m})$

$\mathrm{P}=5.64(1+0,30)$ 
$\mathrm{P}=7.33$ dólares

Valor del margen sobre el precio:

$$
\begin{aligned}
& P=\frac{c}{1-K} \\
& 7.33=\frac{5.64}{1-K} \\
& 1-K=\frac{5.64}{7.33} \\
& K=1-\frac{5.64}{7.33} \\
& K=23.06 \%
\end{aligned}
$$

Para el segundo método se necesita realizar un estudio de mercado que permita establecer una ecuación de la demanda y otra del costo en base de datos histórico o datos proyectados; pero en este caso se supodra datos que permitan demostrar la aplicación de este método.

Este modelo teórico supone que todas las variables se mantienen en el mismo nivel durante el proceso de estudio del efecto del precio sobre las ventas.

Si la función de demanda de un producto es:

$Q=-250 p+3200$

Y la función de costos totales es:

$C=12 Q+11.500$

¿Cuál es el precio que maximiza las utilidades?

Solución: 


\section{BALDEÓN}

$\mathrm{Al}$ examinar las funciones de demanda y costos totales se obtiene:

$$
\begin{aligned}
& A=-250 \\
& K=3.200
\end{aligned}
$$

El remplazar en la expresión (1.10) se tiene:

$$
\begin{aligned}
& p=\frac{v a-K}{2 a} \\
& p=\frac{12(-250)-3200}{2(-250)} \\
& p=12.4 \text { Dólares es el precio óptimo }
\end{aligned}
$$

\section{CONCLUSIONES}

Es fundamental disponer de la información de los costos para establecer el precio, y compararlo con el del mercado para establecer estrategias para que nuestros productos se posicionen en el mercado. Este posicionamiento depende entre otros factores del precio y de la calidad del producto

Tener precios altos o precios bajos sin conocimiento del precio del mercado muchas veces es una decisión de posicionamiento, aunque se trata de una jugada riesgosa, porque no hay reglas a las que atenerse; cada uno lo decide por sí mismo, basándose en su conocimiento del público y el mercado. Una alternativa, arriesgada, es venderla a cinco ocho dólares, pero puede no tener demanda. Otra es venderla a seis, pero entonces por cada sánduche vendido se estará perdiendo un $\$ 1.33$ de utilidad, con respecto al costo de producción, una pérdida que puede resultar muy importante si se pondera por la cantidad de sánduches vendidos al mes. 
ESTRUCTURACIÓN DEL PRECIO DE UN MENÚ

\section{BIBLIOGRAFÍA}

Baca Urbina Gabriel. (2008). Evaluación de Proyectos, Quinta Edición.

Hargadon Bernard J. Jr. y Munera Cárdenas Armando. (2008). Contabilidad de costos.

Cagigal José Luis. (2006). Finanzas de la empresa, Universidad Internacional SEK.

Gómez Bravo Oscar. (2005). Contabilidad de costos, quinta edición. Editorial Mc Graw Hill.

Arboleda Vélez Germán. Proyectos, Formulación, Evaluación y Control. 